\title{
Aplicación de métodos geofísicos al estudio del sistema hidrogeológico Libertad Raigón de Uruguay
}

\author{
Manuel Giménez ${ }^{(1)}$, Agustín Menta(1), Julián Ramos ${ }^{(2)}$, Marcos Musso $^{(1)}$ y Alfonso Flaquer ${ }^{(1)}$ \\ (1) Facultad de Ingeniería - UdelaR. Julio Herrera y Reissig 565, (11300) Montevideo, Uruguay \\ (2) Departamento del Agua - CENUR LN - UdelaR. Rivera 1350 (50000) Salto, Uruguay \\ mgimenez@fing.edu.uy; amenta@fing.edu.uy; jramos@unorte.edu.uy
}

\begin{abstract}
RESUMEN
El Sistema Acuífero Raigón (SAR) es un sistema hidráulico subterráneo en un medio sedimentario que está situado en el departamento de San José, Uruguay. Su rol es fundamental en el abastecimiento de agua a poblaciones y al sistema productivo industrial y agropecuario, siendo el acuífero más explotado del país. El principal objetivo del presente trabajo es caracterizar el contacto entre las Formaciones Libertad y Raigón utilizando dos métodos geofísicos. Por ello se han realizado una serie de perfiles de tomografía de resistividad eléctrica (ERT) y de mediciones mediante sísmica de refracción en cuatro zonas geográficas del SAR donde se dispone de columnas litológicas precisas con diferentes espesores de la Formación Libertad. La comparación entre la información geológica y los modelos geofísicos obtenidos ha permitido calibrar ambos métodos utilizados. Como última etapa de este estudio se seleccionó un quinto emplazamiento con el objetivo de determinar las características del terreno para emplazar nuevos piezómetros de monitoreo. Ambas técnicas geofísicas calibradas han demostrado ser consistentes y complementarias en el estudio del SAR ya que se han logrado asociar rangos de resistividades y de velocidades de propagación de las ondas sísmicas a cada una de las litologías de la Formación Libertad con diferentes espesores, estableciéndose también el contacto con la Formación Raigón.
\end{abstract}

Palabras clave: acuífero Raigón, geofísica, sísmica, tomografía eléctrica.

\section{Application of geophysical methods for the study of the Libertad Raigón hydrogeological system in Uruguay}

\begin{abstract}
The Raigón aquifer system (SAR) is a hydrogeological system in a sedimentary environment, which is located in the department of San José, Uruguay. Its role is fundamental in supplying water for urban, industrial and agricultural uses and it is the most exploited aquifer in the country.

The main objective of this study is to characterize the contact between the Libertad Fm. and Raigón Fm. using two geophysical methods. Therefore, we have carried out a series of profiles of electrical resistivity tomography (ERT) and seismic measurements (refraction) in four geographical areas of the SAR where precise lithological columns are available with different thicknesses of the Libertad Fm. The comparison between the geological information and the geophysical models obtained has allowed both methods to be calibrated. In the last stage of this study, a fifth site was selected in order to determine the characteristics of the land to place new monitoring piezometers. Both geophysical calibrated techniques have proven to be consistent and complementary in the study of the SAR. It was possible to associate ranges of resistivity and the velocity of seismic waves to each of the lithologies of the Libertad Fm with different thicknesses, establishing their contact with the Raigón Fm.
\end{abstract}

Keywords: geophysics, electrical tomography, seismic, Raigón aquifer. 


\section{Introducción}

El Sistema Acuífero Raigón (SAR) es el sistema hidráulico subterráneo más explotado de Uruguay para abastecimiento de agua a poblaciones, actividades industriales y agropecuarias (De los Santos, 1998; De los Santos Gregoraschuck et al., 2019)).

El área de estudio (Figura 1) se ubica en el departamento de San José y representa una de las zonas hidrológicas más productivas del SAR.

En las últimas décadas, debido al incremento de las actividades agrícolas e industriales, el SAR está sometido a una mayor presión desde el punto de vista ambiental.

La Formación Raigón está constituida por areniscas finas a gruesas, conglomerados y niveles arcillosos; tratándose de un acuífero sedimentario de porosidad primaria (Postiglione, M., et al. 2009). Ha sido definido desde libre a semiconfinado, puesto que en un $80 \%$ del área está cubierto por la formación Libertad, que está constituida por limos y arcillas de origen eólico según la caracterización de Goso en 1965 (Ubilla, M. et al. 2004), identificándose principalmente arcillas esmectíticas (Musso et al. 2003).

Los datos piezómetricos muestran que la Formación Libertad constituye una de las principales vías de recarga del acuífero (De los Santos, 1998, Postiglione, M., et al. 2009). Esta hipótesis contrasta con la baja conductividad hidráulica que se les atribuye a los sedimentos que constituyen la Formación Libertad (Dto. Geotécnica, 1997). Determinar el rol que cumple la Formación Libertad en la dinámica de recarga del acuífero, constituye uno de los principales desafíos para los investigadores que estudian el SAR.

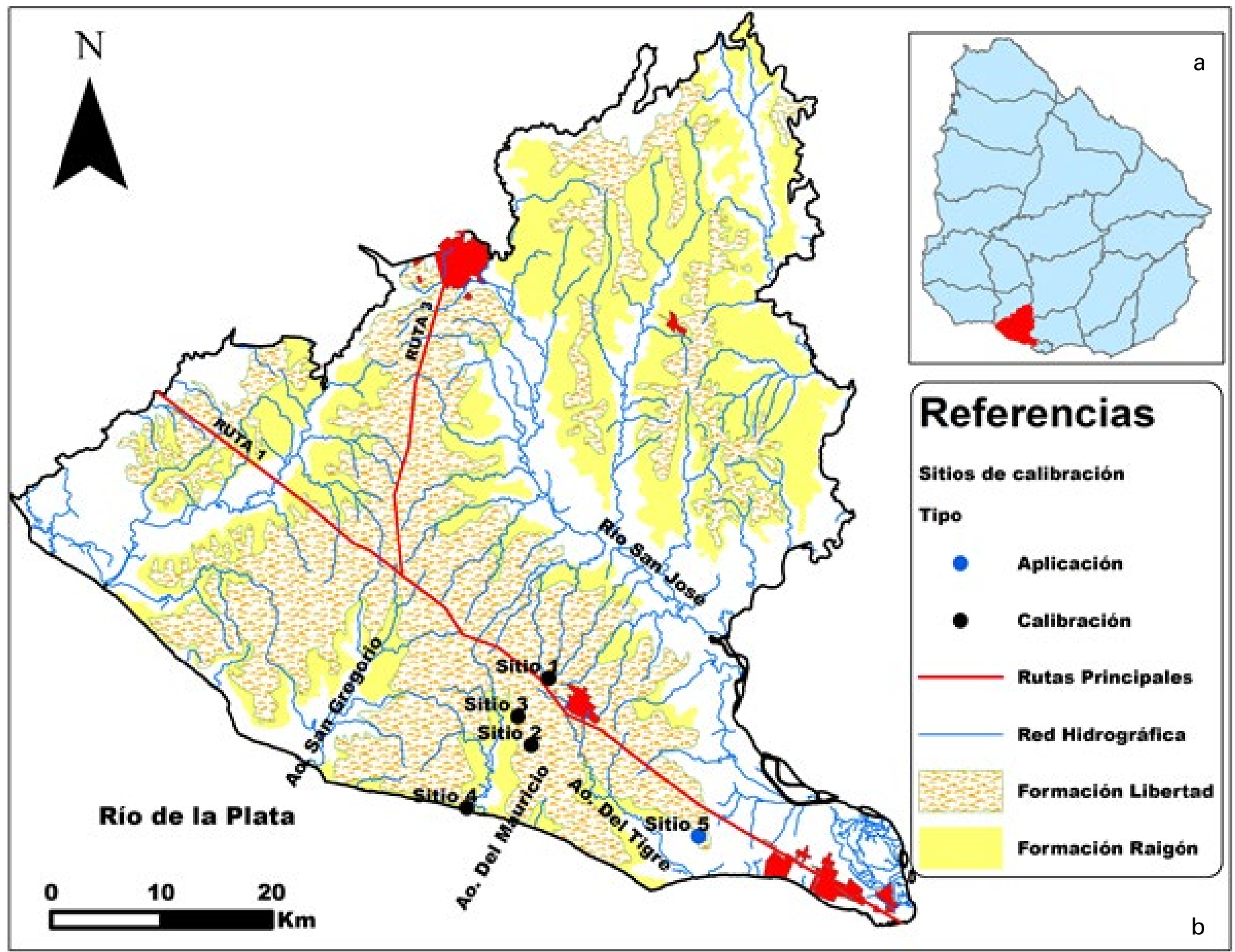

Figura 1. (a) Mapa General de Uruguay con la zona de estudio marcada. (b) Zona de estudio Sistema Acuífero Raigón y sitios de relevamiento geofísico.

Figure 1. (a) General map of Uruguay with the study area marked. (b) Raigón aquifer system study area and the geophysical survey sites. 
Numerosas investigaciones con fines hidrogeológicos han demostrado que los métodos geofísicos constituyen una herramienta complementaria para entender el escenario geológico en el que el agua se encuentra alojada. Comparativamente, los métodos geofísicos son menos invasivos y relativamente más económicos que los métodos hidrogeológicos (OreIlana, 1972, Linde et al., 2006). Entre los métodos utilizados con mayor énfasis en este tipo de investigaciones se encuentran los eléctricos de corriente continua y los sísmicos.

La técnica de la Tomografía de Resistividad Eléctrica (Electrical ResistivityTomography, ERT) en dos dimensiones es un método geofísico en corriente continua que permite investigar de manera continua la variación de la resistividad del subsuelo en profundidad y lateralmente (Griffths and Baker, 1993). La adquisición de datos se realiza mediante un dispositivo compuesto por un resistivímetro, una unidad conmutadora, una computadora, cables de electrodos con varios conectores y electrodos (Dahlin, 2001). Para la interpretación, los datos medidos deben ser invertidos de manera automática mediante la implementación de algoritmos matemáticos, para así obtener un perfil 2D de resistividades (Loke and Barker, 1996).

Por su parte, la sísmica de refracción estudia la propagación de ondas elásticas en el terreno producidas de manera artificial, con el objetivo de determinar la configuración geológica del subsuelo. El método es ampliamente aplicado en la ingeniería de prospección geológica, en disciplinas como la hidrogeología y geotécnica. Consiste en la generación de un tren de ondas mediante un impacto sobre el terreno, con cargas explosivas o dispositivo mecánico de impacto, en distintos puntos relativos de la transecta en la cual se encuentran los geófonos que van a registrar su efecto. Como menciona Reynolds (1997), la refracción sísmica registra la componente refractada de una onda que incide en un límite entre dos medios donde existe contraste de-impedancia acústica y se cumple que la velocidad del segundo medio es mayor que la del primero. El cambio de la dirección de la onda transmitida al segundo medio está gobernado por la Ley de Snell. El arribo de cada onda es detectado a lo largo de una serie de geófonos y registrado en un sismograma. A partir de la interpretación se obtiene un perfil 1D de profundidades y velocidades de ondas $P$.

Existe un gran número de investigaciones que emplean métodos geofísicos de forma conjunta para el estudio de las propiedades del subsuelo; como sísmica de refracción, ERT y GPR (Ground Penetrating Radar) (Doetsch et al., 2012, Amir Gabr et al., 2012). La complementariedad entre los modelos eléctricos y sísmicos permite obtener más información del subsuelo, como pueden ser la distinción entre zonas acuitardas y permeables y sus respectivos estados mecánicos.

Son escasos los estudios en detalle de las Formaciones Libertad y Raigón utilizando técnicas geofísicas.

El objetivo de este trabajo es profundizar en el cono- cimiento del contacto entre las Formaciones Libertad y Raigón mediante el uso combinado del método de exploración eléctrica ERT y de sísmica de refracción de ondas P; para ello se han realizado una serie de perfiles de contraste en lugares donde se conocía la columna litológica que han servido de calibración para extrapolar estas dos técnicas a la exploración de otras zonas dentro del SAR.

\section{Área de estudio}

El área de estudio se localiza al sur de Uruguay, en el departamento de San José. EI SAR (Figura 1b) es un sistema hidráulico subterráneo en un medio sedimentario de origen fluvial a fluviodeltaíca (Preciozzi et al., 1985, Pérez, 1991) que abarca $2271 \mathrm{~km}^{2}$.

Preciozzi et al., (1985) correlacionan la Formación Raigón al Mioceno Superior - Plioceno, mientras que Bossi y Navarro (1988) le asignan edad Pliocena Superior. Según Preciozzi et al., (1985), esta formación está compuesta por areniscas finas a conglomeráticas, mal seleccionadas, con estratificación cruzada y paralela, de color blanco amarillento con intercalaciones de arcillas verdes. Se presenta en parte cubierta por limos arcillosos de las Formaciones Libertad y Dolores y en parte aflorando al norte de la Ruta 1 y al sur en los entalles de los arroyos San Gregorio, Mauricio y delTigre. La Formación Libertad pertenece al Pleistoceno Inferior. Se compone de lodolitas, loess y fangolitas con porcentaje variable de arenas y arcillas, de color pardo a pardo rojizo. Sedimentación continental peridesértica (Preciozzi et al., 1985). Estudios muestran que el contenido de limo y arcillas es del orden de $85 \%$ a $100 \%$ (Musso et al., 2003). El ambiente deposicional es continental, a semiárido con períodos de clima templado húmedo, en el cual se produjeron flujos de barro con arrastre de partículas groseras. El relieve es suavemente ondulado, alcanza cotas de hasta $50 \mathrm{~m}, \mathrm{y}$ una potencia que oscila entre los $2 \mathrm{~m}$ y $35 \mathrm{~m}$. La mineralogía de la fracción arcilla está compuesta por esmectita cálcica dominante, illita secundaria y a veces caolinita (Musso, 2001. Las unidades geológicas pelíticas (limo y arcillas) son reconocidas como unidades con baja conductividad hidráulica (Freeze and Cherry, 1979). La Formación Libertad constituye un techo de baja permeabilidad para la Formación Raigón.

La zona de estudio considerada corresponde al área geográfica ocupada por el SAR, limitándose a las zonas donde la Formación Libertad se encuentra sobreyacente a la Formación Raigón.

\section{Metodología}

En la primera etapa se recopilaron y analizaron los datos y la información existente sobre la zona de estudio: topografía, geología, perfiles litológicos de perforaciones, geofísica e imágenes satelitales. Se analizaron 218 perfiles de perforaciones para determinar la distribución de los espesores de la Formación y se 
seleccionaron cuatro sitios, donde se encuentran perfiles representativos del SAR con diferentes espesores de la Formación Libertad (Figura 1b). En cada uno de estos sitios se cuenta con un pozo con descripción litológica completa, que se ha utilizado para calibrar los métodos geofísicos a implementar.
Se realizaron las campañas de relevamiento de datos geofísicos, utilizando ERT y sísmica de refracción en los cuatro sitios seleccionados en la etapa anterior. Se utilizó un resistivímetro y conmutador de un canal modelo WDJD-3/WDZJ y un sismógrafo WZG 24-A ambos fabricados por WTS/BTSK.


Figura 2. (a) Sismógrafo para las mediciones sísmicas (b) Dispositivo de poleas para las mediciones sísmicas (c) Resistivímetro y línea de electrodos utilizado para las mediciones eléctricas.

Figure 2. (a) Seismograph for seismic measurements. (b) Pulley device for the seismic measurements. (c) Resistivimeter and electrode line used for the electrical measurements. 




Figura 3. Sismograma con primera onda de llegada obtenido para el sitio 1.

Figure 3. Seismogram with first arrival wave obtained for site 1.

En todos los sitios se efectuaron tres perfiles de ERT con 60 electrodos sobre un mismo transepto, con diferente separación entre electrodos: $1 \mathrm{~m}, 3 \mathrm{~m}$ y $6 \mathrm{~m}$. Cada implantación se midió con tres configuraciones electródicas distintas: Wenner Alpha, Wenner Beta y Wenner Gamma. La disposición electródica en estos dispositivos es mostrada por Reynolds (1997). Se obtuvieron 12 perfiles ERT por sitio de exploración (Figura 1a).

Complementariamente, en cada sitio se realizó un perfil sísmico, ejecutando cinco golpes (stacking) en el mismo sitio con el fin de mejorar la relación señal/ ruido. Se utilizaron entre 12 y 17 geófonos, distanciados entre $3 \mathrm{~m}$ y $10 \mathrm{~m}$ según cada sitio. La fuente de energía utilizada consistió en un dispositivo de poleas desarrollado durante el proyecto (Figuras $2 a$ y $2 b$ ), que permitió realizar el impacto sobre el terreno con una masa de $65 \mathrm{~kg}$ y desde una altura estandarizada de 1 metro en todas las mediciones.

Dado que el objetivo era calibrar ambos métodos geofísicos en los sitios con información geológica, se planificó el uso de los diferentes espaciados entre electrodos y geófonos para evaluar los modelos geofísicos obtenidos en cada uno de ellos en términos de resolución horizontal y vertical.

Asimismo, los modelos eléctricos obtenidos con las distintas configuraciones electródicas fueron calibrados a fin de establecer cuál de ellas era la más adecuada para aplicar en sitios desconocidos.

Como última etapa, se seleccionó un quinto sitio de estudio dentro del SAR (Figura 1b) donde aplicar los métodos geofísicos calibrados con el objetivo de determinar las características del terreno para emplazar nuevos piezómetros de monitoreo.
En este caso se realizaron dos perfiles eléctricos utilizando 46 electrodos con separación de $1 \mathrm{~m}$ y 3 $\mathrm{m}$ entre ellos y se optó por la configuración Wenner Gamma. En el mismo emplazamiento se realizaron medidas de velocidades de propagación de ondas $\mathrm{P}$ utilizando la línea sísmica con 12 geófonos separados $5 \mathrm{~m}$ entre sí y un tiro directo distanciado $5 \mathrm{~m}$ del primer geófono.

Los datos eléctricos relevados en campo se interpretaron utilizando el programa comercial de inversión RES2DINVx64 V 4.00 de la firma GEOTOMO (Loke, 2000). En la Tabla 1 se especifican los parámetros más significativos del cálculo para obtener los modelos 2D de resistividad.

\begin{tabular}{|c|c|c|c|c|c|c|c|c|c|c|}
\hline \multirow{2}{*}{ Sitio } & \multirow{2}{*}{$\begin{array}{c}\text { Dispositivo } \\
\text { electródico/ } \\
\text { Espaciado } \\
\text { entre } \\
\text { electrodos }\end{array}$} & \multicolumn{3}{|c|}{$\begin{array}{l}\text { Número de } \\
\text { iteraciones }\end{array}$} & \multicolumn{3}{|c|}{ Error de ajuste (\%) } & \multicolumn{3}{|c|}{$\begin{array}{l}\text { Profundidad } \\
\text { máxima de } \\
\text { exploración }(\mathrm{m})\end{array}$} \\
\hline & & 1 & 3 & 6 & 1 & 3 & 6 & 1 & 3 & 6 \\
\hline \multirow{3}{*}{1} & Alpha & 6 & 4 & 7 & 0.89 & 2.00 & 4.00 & 10 & 28 & 62 \\
\hline & Beta & 6 & 7 & 6 & 0.46 & 0.39 & 0.83 & 9 & 28 & 67 \\
\hline & Gamma & 6 & 5 & 7 & 0.82 & 1.22 & 3.10 & 12 & 36 & 72 \\
\hline \multirow{3}{*}{2} & Alpha & 7 & 4 & 4 & 0.56 & 1.00 & 2.20 & 12 & 35 & 65 \\
\hline & Beta & 7 & 6 & 6 & 0.45 & 0.35 & 0.53 & 9 & 25 & 55 \\
\hline & Gamma & 7 & 5 & 6 & 0.43 & 0.83 & 1.97 & 14 & 37 & 75 \\
\hline \multirow{3}{*}{3} & Alpha & 5 & 6 & 5 & 1.02 & 1.06 & 1.95 & 10 & 31 & 65 \\
\hline & Beta & 7 & 7 & 7 & 0.53 & 0.39 & 0.41 & 9 & 27 & 55 \\
\hline & Gamma & 7 & 6 & 6 & 0.69 & 0.94 & 1.62 & 13 & 36 & 70 \\
\hline \multirow{3}{*}{4} & Alpha & 7 & 6 & 5 & 0.56 & 1.51 & 4.10 & 12 & 35 & 60 \\
\hline & Beta & 7 & 7 & 6 & 0.56 & 0.66 & 1.37 & 9 & 25 & 50 \\
\hline & Gamma & 7 & 4 & 7 & 0.58 & 3.22 & 3.30 & 13 & 37 & 65 \\
\hline
\end{tabular}

Tabla 1. Parámetros más significativos del procesado de los perfiles 2D-ERT.

Table 1. Most significant parameters of 2D-ERT profile processing.

Para el procesamiento de los datos sísmicos se supuso un modelo de capas plano-paralelas horizontales. Se identificaron los tiempos de llegada de la primera onda en cada canal. A continuación, en la Figura 3 , se muestra el sismograma correspondiente al Sitio 1. Con línea roja se marca la primera llegada en cada canal. El canal 14, ubicado en la progresiva 140 se eliminó del procesamiento por ser un canal ruidoso.

En una planilla de cálculo se ingresaron los datos obtenidos y se construyó el hodógrafo (Figura 4). Las velocidades de cada capa se obtuvieron a partir del cálculo del inverso de la pendiente de la ecuación de la línea de tendencia que correlaciona la onda directa y la onda refractada.

El espesor de la capa z se obtuvo mediante la Ecua- 


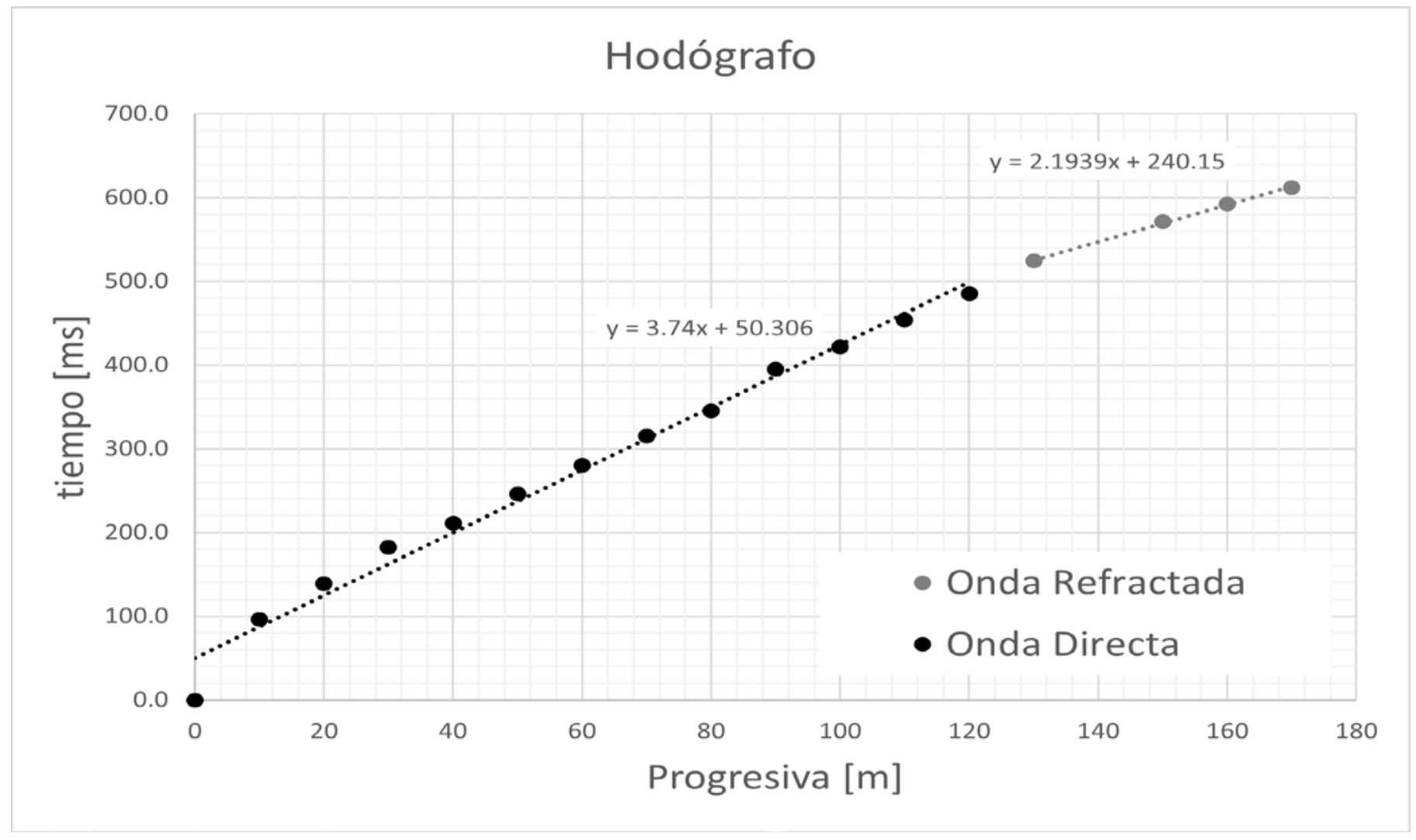

Figura 4. Hodógrafo obtenido para el Sitio 1. Figure 4. Hogograph obtained for site 1.

ción 1 según Reynolds (1997) donde V1 es la velocidad de la onda directa, V2 es la velocidad de la onda refractada y $t_{i}$ refiere al tiempo intercepto que es el tiempo obtenido de la intersección de la dirección de la línea que correlaciona la primera llegada de la onda refractada con el eje de tiempo. Los resultados obtenidos son gráficas (1D) de la velocidad sísmica en función de la profundidad.

En laTabla 2 se incluyen los parámetros más significativos para el procesamiento de los datos sísmicos.

\begin{tabular}{|cccc|}
\hline Sitio & $\begin{array}{c}\text { Número de } \\
\text { Geófonos }\end{array}$ & $\begin{array}{c}\text { Separación } \\
\text { entre } \\
\text { geófonos }(\mathrm{m})\end{array}$ & $\mathrm{t}_{\mathrm{i}}(\mathrm{s})$ \\
\hline 1 & 17 & 10 & 0.24 \\
2 & 12 & 5 & 0.07 \\
3 & 12 & 5 & 0.07 \\
4 & 12 & 3 & 0.02 \\
\hline
\end{tabular}

Tabla 2. Parámetros más significativos del procesado de las secciones 1D-Sísmica de refracción.

Table 2. Most significant parameters of the processing of sections $1 D$ - seismic refraction.

$$
z=t_{i} V_{1} V_{2} / 2\left(V_{2}^{2}-V_{1}^{2}\right)^{1 / 2}
$$

Ecuación 1 (Reynolds, 1997).

\section{Resultados y discusión}

\section{Calibración de los métodos geofísicos}

Tomografías de resistividad eléctrica

En las Figuras 5 a 7, se presentan las nueve secciones de ERT obtenidas a partir de la inversión de los datos para el sitio 1. Todos los perfiles obtenidos, sin importar el dispositivo electródico practicado, muestran una alta consistencia entre sí y un error de ajuste menor al $4 \%$.

La disposición electródica cada $1 \mathrm{~m}$ (Figura 5), alcanzó una profundidad máxima de exploración de 12 $\mathrm{m}$, donde se observa en el primer metro de profundidad una capa resistiva en el entorno de los $13 \mathrm{Ohm} . \mathrm{m}$ y por debajo, una capa de resistividad en el entorno de 5 Ohm.m.

La disposición electródica cada $3 \mathrm{~m}$ (Figura 6) alcanza una profundidad máxima de exploración de $36 \mathrm{~m}$ con un perfil resistivo homogéneo de $5 \mathrm{Ohm}$.m. El dispositivo Wenner Gamma, al alcanzar una mayor profundidad de exploración, denota un aumento gradual de la resistividad a partir de los $32 \mathrm{~m}$ de profundidad.

La disposición electródica cada $6 \mathrm{~m}$ (Figura 7) alcanza una profundidad máxima de investigación de 72 $\mathrm{m}$. En esta sección resistiva se diferencian dos capas, 


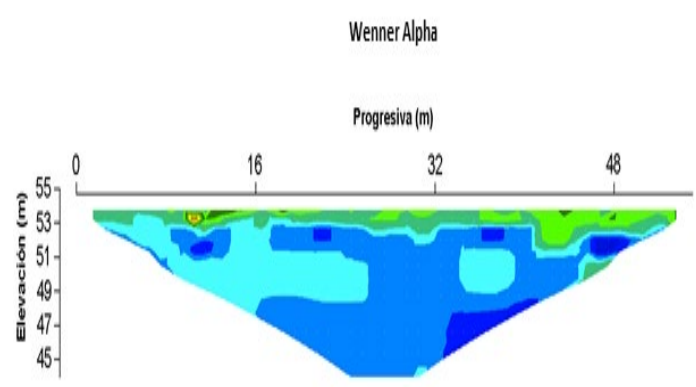

Wenner Beta



Wenner Gamma

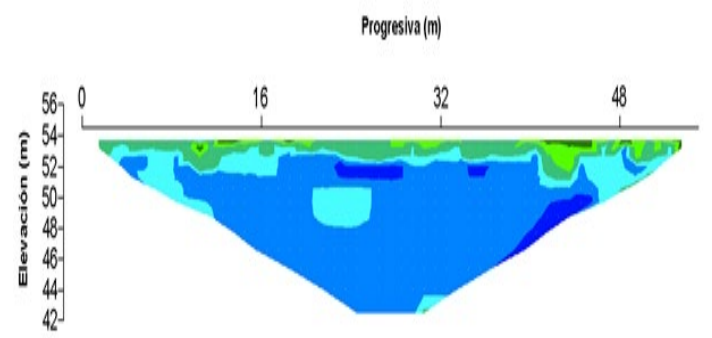

-

$1.03 .0 \quad 5.0 \quad 8.0 \quad 13.017 .025 .035 .0$

RESISTIVIDAD (Ohm.m)
Wenner Alpha

Progresiva (m)

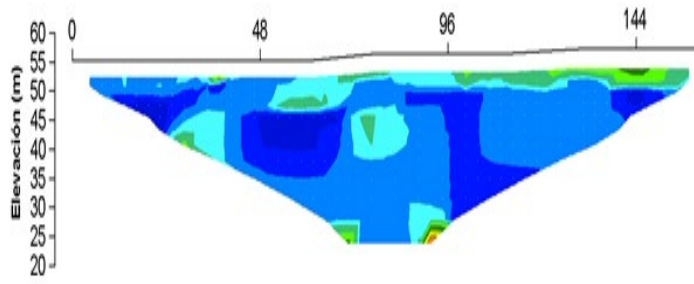

Wenner Beta

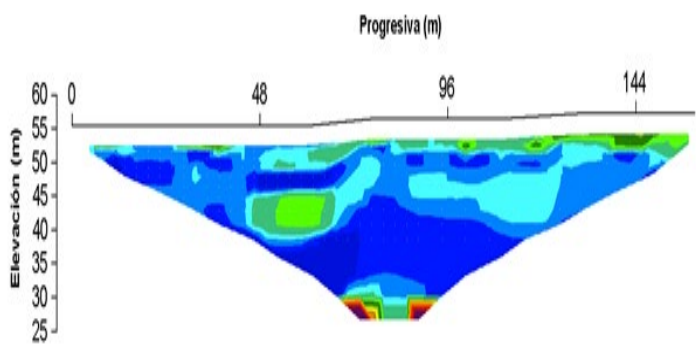

Wenner Gamma

Progresiva (m)

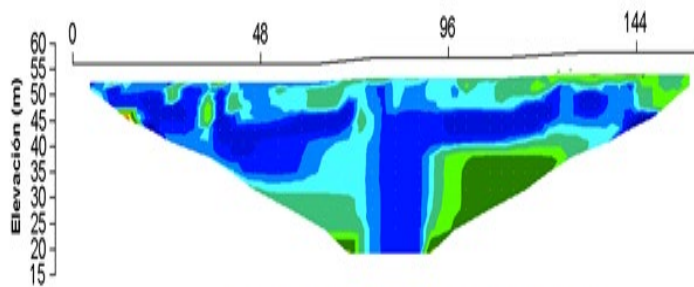

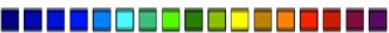

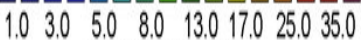
RESISTIVIDAD (Ohm.m)
Figura 5. Secciones de resistividades obtenidas a partir de la inversión de las medidas del Sitio 1 utilizando los dispositivos Wenner Alpha, Wenner Beta, Wenner Gamma con disposición de electrodos cada $1 \mathrm{~m}$. Figure 5. ERT profiles obtained from the inversion of the site 1 measurements using the Wenner Alpha, Wenner Beta, Wenner Gamma devices with an electrode arrangement every $1 \mathrm{~m}$.

la primera con resistividades en el entorno de los 5 Ohm.m y un espesor de $30 \mathrm{~m}$ y una segunda capa a partir de los $38 \mathrm{~m}$ con resistividades en el entorno de los $30 \mathrm{Ohm} . \mathrm{m}$, existiendo una zona de transición entre los $30 \mathrm{~m}$ y $38 \mathrm{~m}$ con un aumento progresivo de la resistividad.

Si bien el error de ajuste del dispositivo Wenner Gamma es mayor que el error de ajuste del dispositivo Wenner Beta en la mayoría de los sitios estudiados, el error en todos los casos no se considera significativo por ser una aplicación hidrogeológica (menor al $4 \%$ ).

El dispositivo Wenner Gamma es el que alcanzó mayor profundidad de investigación para cada disposi-
Figura 6. Secciones de resistividades obtenidas a partir de la inversión de las medidas del Sitio 1 utilizando los dispositivos Wenner Alpha, Wenner Beta, Wenner Gamma con disposición de electrodos cada $3 \mathrm{~m}$. Figure 6. ERT profiles obtained from the inversion of the site 1 measurements using the Wenner Alpha, Wenner Beta, Wenner Gamma devices with an electrode arrangement every $3 \mathrm{~m}$.

ción electródica empleada y los resultados obtenidos no pierden detalle respecto a los otros dispositivos practicados. Por ello se lo considera el más idóneo según los objetivos planteados del estudio.

En la Figura 8 se presenta la sección de resistividad que se considera más representativa (dispositivo Wenner Gamma) para el sitio 1 con separación de electrodos cada $6 \mathrm{~m}$ y el perfil litológico de la perforación existente.

La capa perteneciente a la Formación Libertad que presenta una potencia de 35 metros en el sitio, presenta valores de resistividad en el entorno de 5 Ohm.m. Los valores de resistividad asociados a la capa de la 



Figura 7. Secciones de resistividades obtenidas a partir de la inversión de las medidas del Sitio 1 utilizando los dispositivos Wenner Alpha, Wenner Beta, Wenner Gamma con disposición de electrodos cada $6 \mathrm{~m}$. Figure 7. ERT profiles obtained from the inversion of the site 1 measurements using the Wenner Alpha, Wenner Beta, Wenner Gamma devices with an electrode arrangement every $6 \mathrm{~m}$.
Formación Raigón, son considerablemente mayores y se encuentran en el entorno de los $30 \mathrm{Ohm} . \mathrm{m}$. También se logró identificar el suelo, utilizando la disposición electródica cada 1 metro. La resistividad asociada a esta capa es del entorno de 13 Ohm.m.

Al comparar los resultados con el perfil litológico del pozo, se determina que los primeros $35 \mathrm{~m}$ del perfil corresponden a las arcillas de la Formación Libertad y una resistividad promedio de 5 Ohm.m y la capa subyacente corresponde a las arenas de la Formación Raigón con una resistividad promedio de 30 Ohm.m, evidenciando la zona de contacto entre ambas formaciones.

\section{Sísmica de refracción}

En el Sitio 1 se realizó una medición sísmica con 17 geófonos cada $10 \mathrm{~m}$ debido a la profundidad de investigación requerida para el sitio como establece U.S Army Corps of Engineers (1995). El dispositivo de geófonos y la fuente de energía empleada, permitió alcanzar la profundidad de investigación requerida en el sitio y diferenciar ambas formaciones. Este sitio es donde se encuentran los mayores espesores de la Formación Libertad dentro del área de estudio.

Se muestra a continuación, en la Figura 9, la curva de variación de la velocidad de la onda $P, V p$ en función de la profundidad, para el mencionado sitio.

La velocidad de propagación de ondas $P$ obtenida para la primera capa de $35 \mathrm{~m}$ fue de $200 \mathrm{~m} / \mathrm{s}$, mientras que para la capa inferior se obtuvo una velocidad de propagación de onda $P$ de $700 \mathrm{~m} / \mathrm{s}$. Al comparar estos resultados con el perfil litológico del pozo, se determina que los primeros $35 \mathrm{~m}$ del perfil corresponden a las arcillas de la Formación Libertad y la capa subyacente corresponde a las arenas de la Formación Raigón, evidenciando la zona de contacto entre ambas formaciones.

\section{Complementariedad de los métodos utilizados}

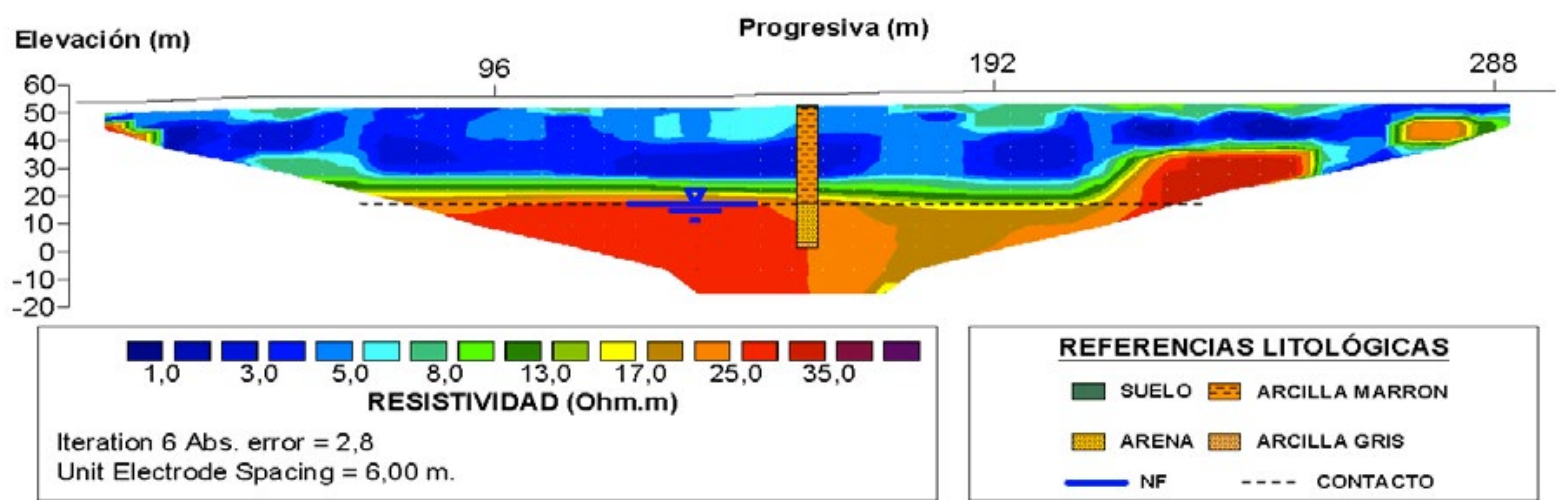

Figura 8. Sección de resistividades obtenida a partir de la inversión de las medidas del Sitio 1 utilizando un dispositivo Wenner Gamma con disposición de electrodos cada 6 metros y perfil litológico del pozo de calibración.

Figure 8. ERT profiles obtained from the inversion of the measurements of site 1 using a Wenner Gamma device with an electrode arrangement every 6 metres and lithological profile of the calibration well. 
En la Tabla 3 se muestra un resumen de los resultados obtenidos en ambos métodos geofísicos para cada uno de los cuatro sitios de calibración usando los modelos eléctricos (2D) provenientes del arreglo Wenner-Gamma con un espaciado entre electrodos de $6 \mathrm{~m}$ y los modelos 1D de velocidades sísmicas obtenidos con un espaciado entre geófonos entre $3 \mathrm{~m}$ y $10 \mathrm{~m}$ para los sitios.

La comparativa entre los perfiles de resistividad y de velocidad de la onda $\mathrm{P}$ presenta una buena correlación entre sí y con la información litológica de cada sitio.

El análisis de las cuatro zonas de calibración indica que los valores de resistividad aparente para la



Figura 9. Velocidad de propagación de la onda P en función de la profundidad para el sitio 1.

Figure 9. $P$ wave propagation velocity as a function of depth for site 1.

Formación Libertad varían entre $5 \mathrm{Ohm} . \mathrm{m}$ hasta 17 Ohm.m, mientras que los valores obtenidos para la Formación Raigón, varían entre 15 Ohm.m hasta 35 Ohm.m, y en algún caso es mayor. Estos rangos distintos de resistividad permiten identificar el contacto entre la Formación Libertad y la Formación Raigón.

La Formación Libertad presenta valores de resistividad que se encuentra dentro del rango indicado por Orellana (1972) para materiales como limos $(20$ Ohm.m a 100 Ohm.m) y arcillas (1 Ohm.m a 100 Ohm.m).

En los sitios donde la Formación Libertad tiene las mayores potencias, los valores de resistividad asociada a la misma son más homogéneos y menores respecto a los sitios donde los espesores de la Formación Libertad son inferiores. Esto es coherente con la teoría geoeléctrica, debido a que capas con mayor espesor permitirán obtener valores de resistividades más cercanos a los específicos del material litológico.

Los valores de resistividad obtenidos para la For- mación Raigón se encuentran dentro de los valores esperados para la composición litológica de esta formación. Según Orellana (1972) las arenas presentan rangos de resistividad desde 100 Ohm.m a 1000 Ohm.m, mayores a los obtenidos en esta investigación. Sin embargo, Astier (1975) hace hincapié en que estos valores pueden variar según el grado de humedad y contenido de arcilla que presenta. En todos los sitios, la Formación Raigón se encuentra en estado de saturación a excepción del Sitio 4 donde el nivel freático se encuentra por debajo de la profundidad investigada.

Los valores de velocidad de propagación de onda $P$ para la Formación Libertad en encuentran entre 200 $\mathrm{m} / \mathrm{s}$ y $400 \mathrm{~m} / \mathrm{s}$. Estos valores son consistentes con Reynolds (1997) que indica que los materiales loessicos presentan velocidades de propagación de ondas $P$ entre $300 \mathrm{~m} / \mathrm{s}$ y $600 \mathrm{~m} / \mathrm{s}$.

Para la Formación Raigón, los valores de velocidad de propagación de ondas $\mathrm{P}$, presentan un mayor rango, desde 700 m/s hasta 2000 m/s. Según Reynolds (1997) los rangos de velocidad de propagación de ondas $P$ para materiales arenosos tienen un mayor rango de variación y pueden ir desde $200 \mathrm{~m} / \mathrm{s}$ para arena suelta hasta $2300 \mathrm{~m} / \mathrm{s}$ para arena y grava cerca de la superficie.

En los sitios estudiados no se puede realizar una correlación directa entre los valores obtenidos de velocidad de propagación de ondas $\mathrm{P}$ para la Formación Raigón y diferentes factores que pueden afectar estos valores como la profundidad de exploración, el grado de saturación y la presencia de arcillas como indica Reynolds (1997). Hay que indicar que los puntos relevados son escasos para poder realizar un estudio estadístico y realizar análisis de correlación con los factores antes mencionados. Sin embargo, los valores obtenidos de velocidad de propagación de ondas P para la Formación Libertad y la Formación Raigón, distos entre sí, permiten diferenciar ambas formaciones a partir de la medición de refracción sísmica.

\section{Resultados en el Sitio 5}

A partir de los resultados de ERT obtenidos en el Sitio 5 , en los primeros $8 \mathrm{~m}$ (entre la cota $26 \mathrm{~m}$ y la cota 18 $\mathrm{m})$ se observan valores de resistividades menores a 8 Ohm.m. A partir de la cota $18 \mathrm{~m}$, los valores de resistividad son mayores a $25 \mathrm{Ohm}$.m. Estos valores son muy similares a los obtenidos en los sitios de calibración, obteniéndose valores de resistividades eléctricas entre 5 y 13 Ohm.m para la Formación Libertad y entre 17 y 35 Ohm.m para la Formación Raigón.

Adicionalmente, en el Sitio 5 se realizó una medición sísmica con 12 geófonos separados $5 \mathrm{~m}$ entre ellos y un tiro directo separado $5 \mathrm{~m}$ desde el primer geófono. Los valores de velocidad de propagación de la onda $\mathrm{P}$ obtenidos, fueron de $200 \mathrm{~m} / \mathrm{s}$ para la Formación Libertad y de 1400 m/s para la Formación Raigón. El cambio de medio se observa a una profundidad aproximada de $8 \mathrm{~m}$, desde el nivel de la cota de terreno. 


\begin{tabular}{|lccc|}
\hline Resultados & Sitio 1 & Sitio 2 & Sitio 3 \\
\hline Espesor Litológico de Formación Libertad (m) & 35 & 6 & 14 \\
Resistividad de Formación Libertad (Ohm.m) & 5 & 17 & 5 \\
Espesor de Formación Libertad con ERT (m) & 35 & 6 & 15 \\
Resistividad de Formación Raigón (Ohm.m) & 30 & $15-35$ & $20-30$ \\
Velocidad Vp Formación Libertad (m/s) & 200 & 200 & 400 \\
Espesor de Formación Libertad con sísmica (m) & 35 & 7 & 15 \\
Velocidad Vp de Formación Raigón (m/s) & 700 & 1100 & 2000 \\
\hline
\end{tabular}

Tabla 3. Resultados obtenidos para los sitios de calibración.

Table 3. Results obtained for the calibration sites.

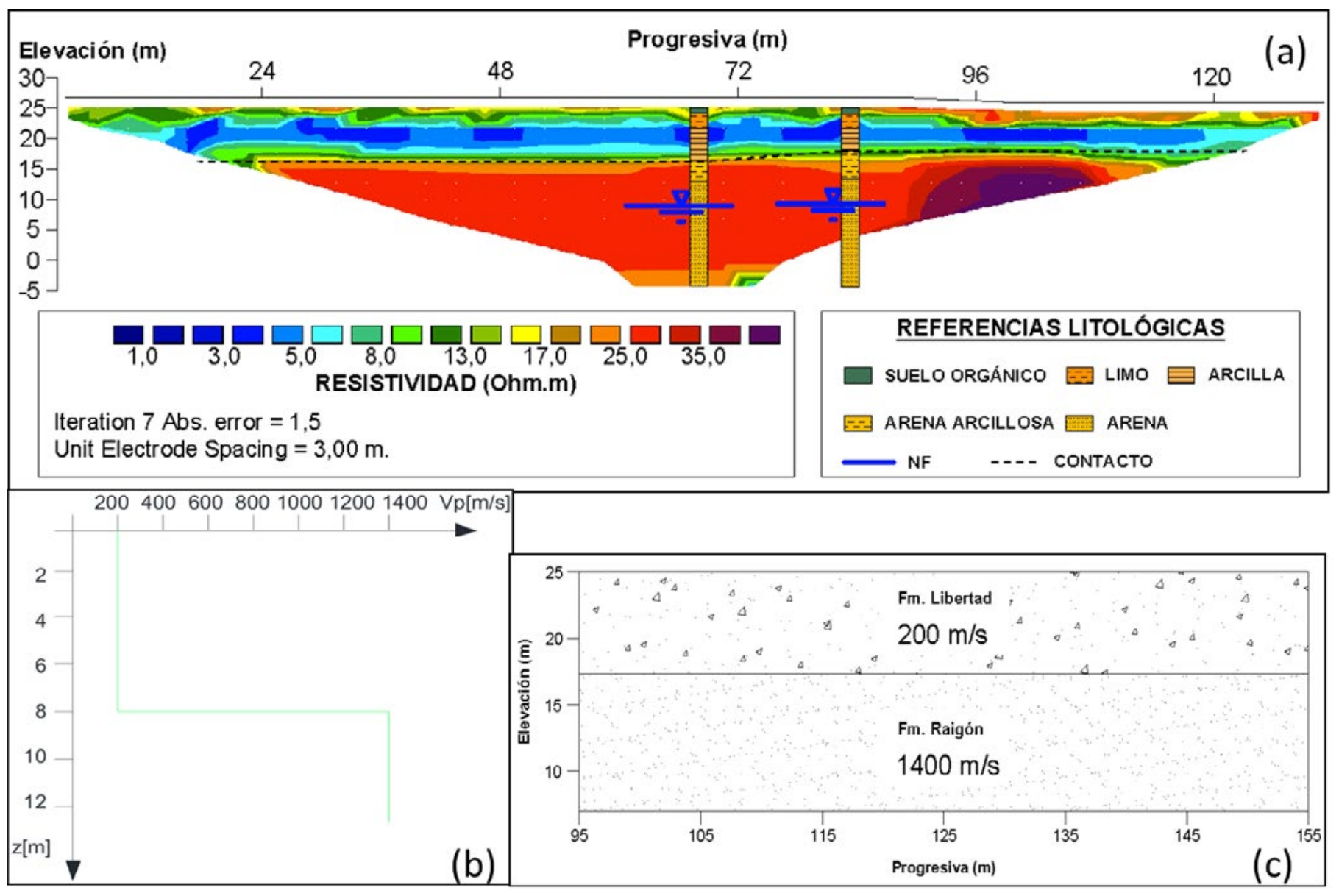

Figura 10. (a) Sección de resistividades obtenida a partir de la inversión de las medidas del Sitio 5 utilizando un arreglo Wenner Gamma con disposición de electrodos cada 3 metros y perfiles litológicos de los piezómetros construidos. (b) Curva espacio-tiempo. (c) Perfil geológico interpretado a partir la medición sísmica.

Figure 10. (a) ERT profiles obtained from the inversion of the site 5 measurements using a Wenner Gamma array with an electrode arrangement every 3 metres and lithological profiles of the piezometers constructed. (b) Space-time curve. (c) Geological profile interpreted from the seismic measurement. 
En la Figura 10 se presentan los resultados obtenidos de ERT junto a los piezómetros construidos en el sitio (Figura 10a). La Figura 10b muestra la curva espacio-tiempo para la medición sísmica y la Figura 10c muestra un perfil geológico interpretado a partir de la medición sísmica y el perfil litológico del sitio.

El perfil litológico interpretado a través de las mediciones de ERT y sísmicas son concordante con lo observado a través del perfil litológico de las perforaciones posteriormente realizadas.

\section{Conclusiones}

En el SAR es posible distinguir ambas formaciones geológicas (Formación Libertad y Formación Raigón) a partir de valores de resistividad y velocidades de propagación de la onda P. Los valores de resistividad aparente para la Formación Libertad se encuentran entre 5 y 17 Ohm.m, mientras que para la Formación Raigón se encuentran entre 15 y 35 Ohm.m. Los valores de velocidad de propagación de ondas sísmicas para la Formación Libertad se encuentran entre 200 m/s y 400 m/s, en tanto para la Formación Raigón variaron $700 \mathrm{~m} / \mathrm{s}$ y $2000 \mathrm{~m} / \mathrm{s}$.

Las prospecciones mediante el método sísmico de refracción permitieron diferenciar correctamente ambas formaciones y detectar la zona de contacto, mientras que la ERT permitió determinar heterogeneidades en los perfiles estudiados que las prospecciones sísmicas, mediante los arreglos empleados, no permitieron observar. Sin embargo, el método sísmico empleado mostró un buen contraste entre ambas formaciones.

Desde el punto de vista operativo y con estos dispositivos empleados, se encuentra que la prospección sísmica resulta un método rápido y sencillo de aplicar mediante los arreglos empleados para el rango de profundidades objetivo a explorar en la zona del SAR. Los tiempos de montaje y medición, mediante este método, son menores respecto a la ERT con esta disposición electródica. Sin embargo, la ERT permite determinar heterogeneidades en el perfil estudiado y una mayor extensión de investigación.

La aplicación combinada de ambos métodos geofísicos, permitió diferenciar correctamente las dos unidades geológicas principales que componen el SAR en un sitio sin perfiles litológicos conocidos. Se ha calibrado una metodología de prospección geofísica para la zona del SAR, que permitirá obtener resultados satisfactorios en otras zonas del acuífero que no se cuenta con información litológica

\section{Agradecimientos}

A la Comisión Sectorial de Investigación Científica de la Universidad de la Republica, por financiar la investigación a través del Programa de Iniciación a la Investigación. Asimismo, los autores agradecen al Grupo de Hidrología Subterránea del Instituto de Mecánica de los Fluidos de la Facultad de Ingeniería y al Departamento del Agua del Centro Regional Universitario del Litoral Norte, pertenecientes a la Universidad de la República de Uruguay, por proveer el equipamiento y recursos humanos y materiales para llevar adelante la investigación.

\section{Referencias}

Amir Gabr et al. 2012. Water resources and wetlands, Editors: Petre Gâștescu, William Lewis Jr., Petre Breţcan Conference Proceedings, 14-16 September 2012, Tulcea - Romania ISBN: 978-606-605-038-8

Astier, J.L. 1975. Geofísica aplicada a la Hidrogeología. Paraninfo. Madrid. 344 p.

Bossi, J. and Navarro, R. 1988. Geología del Uruguay. 2T. Universidad de la República. Montevideo. To$\operatorname{mos} 1+2,970 \mathrm{pp}$.

Departamento de Geotécnica. 1997. Carta Geotécnica de la región Metropolitana de Montevideo escala 1:100.000. Oficina de Publicaciones del Centro de Estudiantes de Ingeniería, $32 \mathrm{pp}$. Montevideo.

De los Santos, J. 1998. Modelación numérica del acuífero de Raigón. Proyecto CONICYT-BID 71/94. Montevideo: IMFIA, Universidad de la República, Uruguay, $80 \mathrm{pp}$.

De los Santos Gregoraschuk, J.E., Custodio Gimena, E., Flaquer Barrios, A.N., Giménez Martínez, M.C., Menta Romano, A., Bessouat Ferraris, C. 2019. Gestión ambiental del sistema acuífero Raigón, Proyecto TPTA 307 (07-2014 a 02-2017). IMFIA, Facultad de Ingeniería. Universidad de la República. Instituto Nacional de Investigación Agropecuaria. Montevideo. Uruguay: 1- 48.

Dahlin, T. 2001. The development of $D C$ resistivity imaging techniques. Computers \& Geosciences, 27(9), 1019-1029.

Doetsch, J. et al., 2012. Constraining 3-D electrical resistance tomography with GPR reflection data for improved aquifer characterization. Journal of Applied Geophysics, 78: 68-76

Freeze, R.A. and Cherry, J.A. 1979. Groundwater. Prentice-Hall Inc., Englewood Cliffs, Vol. 7632, 604.

Linde, N. et al., 2006. Improved hydrogeophysical characterization using joint inversion of cross hole electrical resistance and ground-penetrating radar traveltime data. Water Resources Research, 42(12).

Loke, M.H. and Barker, R.D. 1996. Rapid least-squares inversion of apparent resistivity pseudosections by a quasi-Newton method. Geophysical Prospecting44 (1), 131-152.

Locke, M. H. 2000. RES2DINV, Rapid 2D resistivity and IP inversion (computer program). Distributed by IDS Scintrex, Concord Ontario, Canada.

Musso, M. et al., 2003. Subrasantes expansivas en Rutas Nacionales: ¿mito o realidad?, $4^{\circ}$ Congreso de Ingeniería Vial, Montevideo, Uruguay. 
Musso, M. 2014. Guidelines to identify and quantify expansive soils in civil infrastructure, IAEG XII Congress, Torino, Italy.

Orellana, E. 1972. Prospección geoeléctrica en corriente continua, Paraninfo, Madrid, ISBN: 84-2831153-6.

Pérez, M. 1991. Litoestratigrafía y Sedimentodología de las Formaciones Raigón y Libertad, Suroeste del departamento de San José (Uruguay). Tesis de maestría en Ciencias de la Tierra, Universidad de Montreal en Quèbec, Canadá.

Postiglione, M. et al. 2009. "Gestión sostenible del acuífero Raigón, Uruguay." IAEA, Estudios de hi- drología isotópica en América Latina (2009): 195218.

Preciozzi, F. 1985. Memoria explicativa de la carta geológica del Uruguay a escala 1:500.000.

Reynolds, J. M. 1997. An introduction to applied and environmental geophysics. 1st edition, ISBN 0-47196802-1. John Wiley \& Sons LTD. England, $796 p$

Ubilla, M. et al. 2004. Cuencas Sedimentarias de Uruguay -El Cenozoico. DIRAC-Facultad de Ciencias, pp. $11-36$.

U.S. Army Corps of Engineers. 1995. Geophysical Exploration for Engineering and Environmental Investigations, Washington D.C.,U.S.A. 